\title{
Vélocimétrie Doppler ultrasonore Applications à l'expérimentation et au diagnostic
}

\author{
Ultrasonic Doppler velocimetry \\ Experimental applications and for diagnosis
}

\author{
Pierre Peronneau \\ Maître de Recherche à l'INSERM \\ Instrumentation \& Dynamique cardiovasculaire, ERA CNRS N`070785 \\ Hôpital Broussais, 26, rue Didot, 75674 Paris, Cedex 14
}

\section{Introduction}

La mesure des vélocités sanguines ou du débit est un terme fondamental de toute étude hémodynamique en expérimentation ou en pratique clinique chirurgicale et médicale. En fait, peu de méthodes sont disponibles pour de telles mesures, surtout lorsque l'on désire avoir accès aux grandeurs instantanées afin de suivre leurs évolutions au cours du cycle cardiaque, ce qui nécessite une bande passante minimum de reproduction d'environ vingt-cinq Hertz. Nous citerons essentiellement la débitmétrie électromagnétique, l'anémométrie à films chauds, les méthodes ultrasonores et pour certaines expérimentations sur modèles, l'anémométrie laser et les techniques polarographiques.

Parmi ces techniques, les méthodes ultrasonores Doppler sont apparues comme particulièrement intéressantes. Elles sont basées sur la rétro-diffusion d'une onde ultrasonore par les globules rouges dont le mouvement induit un effet Doppler. Le schéma de principe général est donné par la figure 1. Le capteur comprenant les transducteurs émetteur et récepteur (qui peuvent être confondus) illumine en incidence oblique le territoire à examiner. La fréquence de l'onde rétro-diffusée est modifiée par rapport à celle de l'onde incidente d'une quantité proportionnelle à la vélocité des érythrocytes.

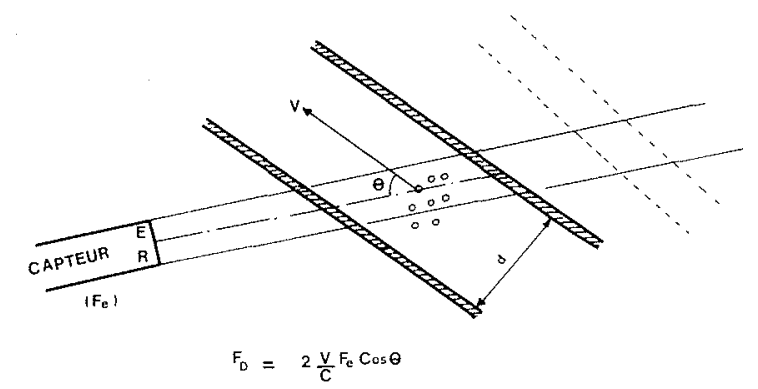

Figure 1 - Principe de la vélocimétrie Doppler ultrasonore.
On appelle communément fréquence Doppler $F_{D}$ la différence entre la fréquence émise et la fréquence reçue. Cette différence est positive ou négative selon le sens de l'écoulement et la détection de son signe peut donc permettre une détection directionnelle de l'écoulement.

En fait, la vélocimétrie Doppler se présente comme une méthode semi-indirecte de la mesure de la vélocité du sang, mais avec une propriété très intéressante : les caractéristiques de propagation des ondes ultrasonores à travers les tissus et le sang permettent des mesures sans interférence avec l'écoulement sur des vaisseaux intacts et même dans nombre de cas, de façon transcutanée.

$\mathrm{Du}$ point de vue technique, le développement de toutes les méthodes Doppler n'est pas encore complètement achevé, mais dès à présent un large éventail d'applications et de possibilités est offert aussi bien pour les applications cliniques que les expérimentations en hémodynamique. Après avoir décrit le principe des appareils utilisés, nous en donnerons quelques exemples.

\section{Appareillages utilisés}

Deux grandes lignes d'appareils sont actuellement développés : les appareils à émission continue et ceux à émission pulsée.

\section{Vélocimètres à émission continue}

\section{Appareils non directionnels}

Ce fut le premier type d'appareil développé $[1,2]$. Le schéma de principe est donné sur la figure 2 (circuit selon A). 


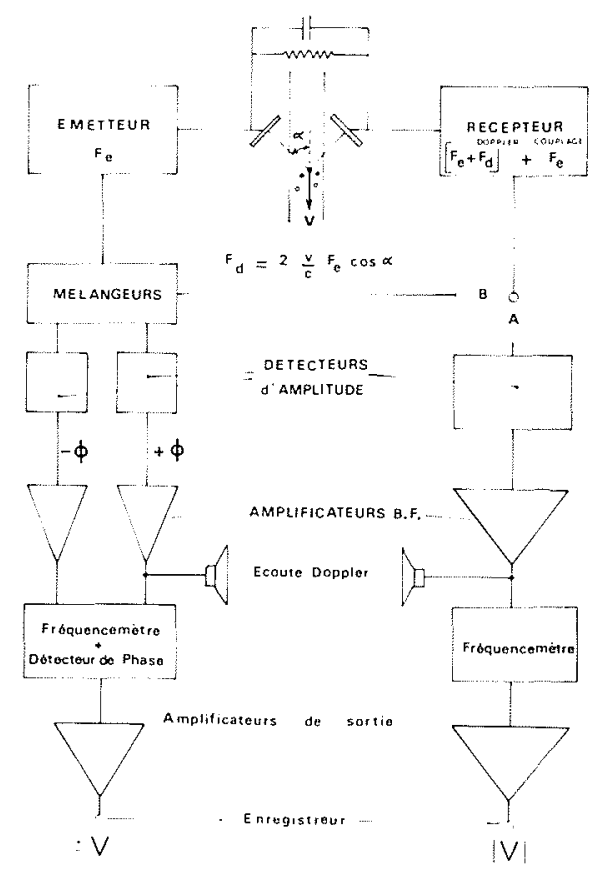

Figure 2 - Schéma de principe des appareils Doppler à émission continue.

circuit $\mathrm{A}:$ non directionnel

circuit $B$ : directionnel

Les signaux Doppler apparaissent par battement entre les fréquences émise et reçue. Le signal de battement, après détection d'amplitude et filtrage, correspond à la valeur absolue de la dérive Doppler. Un filtre passehaut est introduit pour rejeter les échos correspondant aux structures fixes ou mobiles à faible vitesse comme les battements de paroi vasculaire.

Le signal Doppler ainsi isolé est situé dans la gamme audiofréquences et est dirigé sur un haut-parleur qui permet de procéder à une analyse spectrale auditive très riche en renseignements qualitatifs, sur un fréquencemètre ou sur un analyseur de fréquences.

Ce type d'appareil présente de graves défauts concernant la précision des mesures, notamment la méconnaissance du sens de l'écoulement, ce qui est un lourd handicap pour les mesures sur des écoulements pulsatiles.

Cependant, son extrême simplicité le rend précieux pour une auscultation vasculaire, pour des mesures où les vélocités locales sont connues ne pas s'inverser au cours du cycle cardiaque, et enfin pour des implantations chroniques avec télémétrie.

\section{Appareils directionnels}

Des systèmes plus élaborés de détection des signaux Doppler permettant une détection directionnelle de l'écoulement sont maintenant très généralement utilisés (oscillateur de référence à fréquence décalée ou à phase décalée selon l'exemple donné par le circuit $B$ de la figure 2) $-[3,4,5,6]$.

Figure 3. - Principe de la vélocimétrie à émission pulsée

\section{Ambiguités en distance}

Très schématiquement, le capteur illuminant le vaisseau à étudier est défini par son diagramme de rayonnement, c'est-à-dire grossièrement par un faisceau légèrement conique issu de sa face de sortie.

Tout mouvement de paroi ou de globules rouges coupant le faisceau sera perçu. L'information disponible correspondra à une sorte de moyenne des différents mouvements avec une pondération due à l'atténuation de l'onde ultrasonore en fonction de la distance. Ainsi, par exemple, dans la configuration de la figure 1, les informations en provenance des vaisseaux $A$ et $B$ seront mélangées.

\section{Appareils à émission pulsée}

Ce type d'appareillage a été créé pour répondre au besoin de discrimination en distance. C'est la combinaison d'un système échographique et d'un système Doppler (directionnel). C'est un choix particulier parmi les systèmes à émission codée $[7,8,9]$.

\section{Principe}

Le capteur est incliné en direction de l'axe du vaisseau à étudier (Fig. 3). Il est alimenté en impulsions (durée $\tau_{1}$, fréquence $F_{e}$ ). Au niveau des différents interfaces situés sur le faisceau ultrasonore, il y a réflexion partielle d'énergie ultrasonore. Entre deux impulsions, le capteur reçoit les échos correspondant à la paroi vasculaire et aux globules rouges. Une porte électronique permet de sélectionner un volume de mesure réglable en largeur et en position par rapport au capteur, volume dans lequel l'information de vélocité est extraite.

Quelques remarques peuvent être faites:

a) une durée de porte d'observation égale à la période de répétition des impulsions donne une caractéristique spatiale assez similaire à celle d'un appareil continu et peut aider à la recherche du vaisseau ;

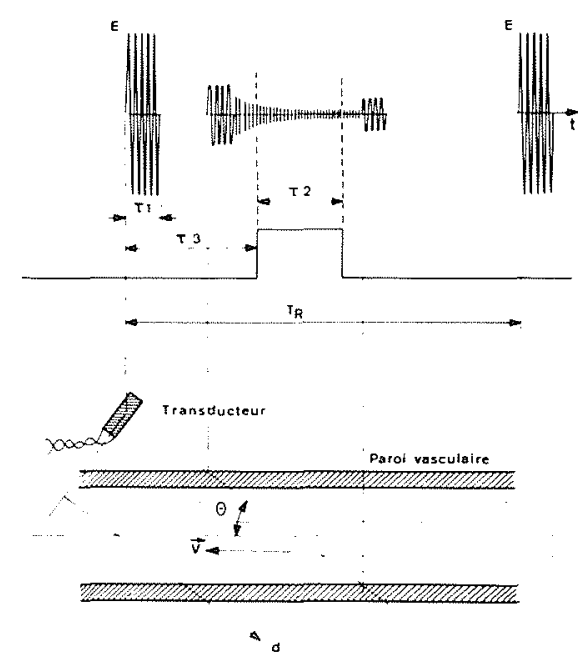


b) une durée très faible de la porte d'observation permet le recueil des vélocités locales, la détermination de la position des parois vasculaires (et l'estimation du diamètre vasculaire) et le relevé point par point de la distribution des vélocités le long d'un diamètre vasculaire. L'acquisition de cette répartition des vélocités le long d'un diamètre peut se faire en temps réel par un système possédant une série de fenetres d'observation simultanées $[10,11]$;

c) la combinaison de la mesure du diamètre vasculaire et de la mesure de vélocité moyenne sur la lumière vasculaire conduit à une mesure du débit volumique.

Le schéma synoptique d'un vélocimètre à émission pulsée est donné sur la figure 4.

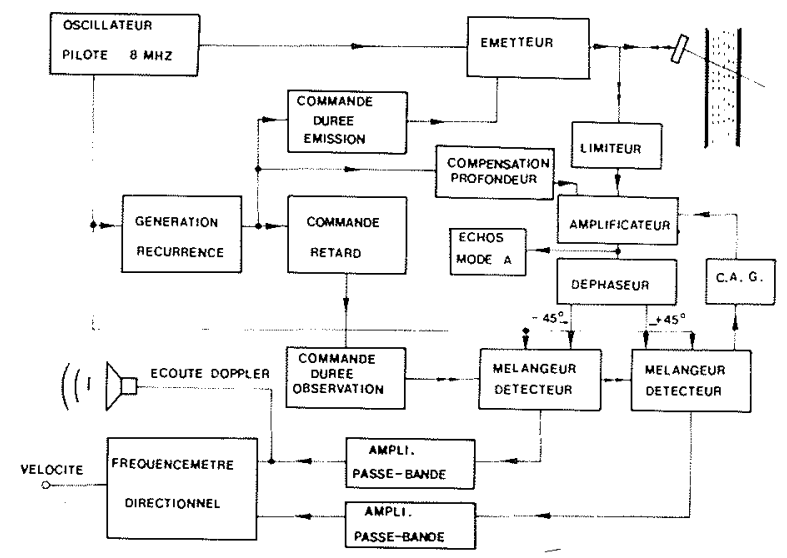

Figure 4 - Schéma de principe d'un vélocimètre à émission pulsée

\section{Ambiguités}

Des ambiguités dans le repérage des distances peuvent se produire puisque l'on utilise un processus d'exploration récurrent; pour la même raison, une ambiguité sur la mesure de vélocité peut exister [11]. La distance maximale et la vélocité maximale que l'on peut mesurer sans ambiguité sont reliées par (dans la direction du faisceau ultrasonore) :

$$
\mathrm{d}_{\max } \times \mathrm{v}_{\max }=C^{2} / 8 F_{e}
$$

Numériquement pour $F_{e}=4 \mathrm{MHz}, \theta=45^{\circ}$,

$$
\mathrm{d}_{\max }(\mathrm{cm}) \times \mathrm{v}_{\max }(\mathrm{cm} / \mathrm{s})=1000
$$

\section{Volume de mesure}

Le volume de mesure est déterminé par la durée de la porte d'observation, mais également par la durée des impulsions émises; par les dimensions latérales du faisceau et la bande passante du capteur et de l'appareillage. La résolution du système est déterminée par la plus petite dimension réalisable pour le volume de mesure $[10,11]$. Elle dépend du type de mesure effectué mais typiquement s'établit entre 0,5 et $1,5 \mathrm{~mm}$ (dans la direction du faisceau). Lorsque une grande précision est nécessaire, différentes procédures de correction peuvent être appliquées $[10,12]$.
Analyse et mesure des fréquences Doppler

Quel que soit le principe de l'appareil utilisé pour l'exploration d'un territoire vasculaire, il fournit un signal Doppler qu'il s'agit de traiter pour obtenir des informations de vélocité proprement dite. De nombreux problèmes se posent pour l'exploitation du signal Doppler et différents systèmes de traitement sont utilisés [13].

\section{Complexité de la mesure de vélocité}

Elle est due principalement au grand nombre de cibles participant à l'information Doppler : ces cibles sont constituées par les globules rouges et donc animées de vélocités différentes et variables au cours du temps. L'information Doppler est ainsi constituée par un spectre de fréquences $(P(f))$ évolutif au cours du temps. Moyennant certaines hypothèses, on peut montrer que la vélocité moyenne (dans le volume de mesure) est donnée par :

$$
\bar{v}=k \frac{\int_{-\infty}^{+\infty} f P(f) d f}{\int_{-\infty}^{+\infty} P(f) d f}
$$

Le mouvement éventuel des structures adjacentes au vaisseau, et de toute manière le battement de la paroi artérielle induisent des signaux Doppler de haute énergie comparativement aux signaux induits par les globules rouges, mais de fréquence faible. On réduit cette interférence par un filtrage approprié, mais qui élimine alors la possibilité de mesurer des vélocités d'écoulement très faibles (de l'ordre du $\mathrm{cm} / \mathrm{s}$ ).

\section{Fréquencemètres}

Ce sont les dispositifs convertissant le signal Doppler en un signal de vélocité, idéalement $\bar{\nu}$.

\section{Compteur de passages à zéro du signal Doppler}

C'est le dispositif le plus simple et le plus généralement utilisé. Avec quelques hypothèses, on peut montrer que sa réponse est donnée par :

$$
\bar{v}^{\prime}=k \sqrt{\frac{\int_{-\infty}^{+\infty} f^{2} P(f) d f}{\int_{-\infty}^{+\infty} P(f) d f}}
$$

Une erreur est donc commise $\left.\overline{\left(v^{\prime}\right.}-\bar{\nu}\right)$ qui dépend du régime d'écoulement.

Le signal Doppler comporte une composante de bruit qui est négligeable lorsque le rapport signal à bruit est suffisant $(20 \mathrm{~dB})$. Cependant autour d'un écoulement très faible, ou nul, le fréquencemètre tend à donner une information relative au bruit puisque dans cette condition les fréquences Doppler sont très faibles ; on est amené à introduire un seuil pour éviter un "comptage 
du bruit". Il peut en résulter une perte de sensibilité et une distorsion si le rapport signal à seuil n'est pas suffisant.

\section{Fréquencemètres donnant une vélocité moyenne}

De tels dispositifs ont été réalisés au moins sous forme analogique ; relativement complexes, en tout cas par rapport aux compteurs de passages à zéro, ils sont encore très peu utilisés en pratique courante [13].

\section{Analyse spectrale}

L'information Doppler recueillie avec un vélocimètre ultrasonore est plus riche que simplement la donnée de fréquence/vélocité moyenne, et permet d'accéder au moins qualitativement à des caractéristiques de l'écoulement $[12,13,14]$. Pour cela on effectue une analyse spectrale du signal Doppler très grossièrement à l'oreille (en écoutant le spectre Doppler par l'intermédiaire d'un haut-parleur) ou par un analyseur de spectre. Il est particulièrement souhaitable de disposer des résultats de l'analyse en temps réel au cours de l'examen. Plusieurs types de dispositifs ont été développés : banque de filtres, système à mémoire circulante, transformateur de Fourier rapide, histogramme des passages à zéro du signal Doppler. Au-delà des problèmes posés par une présentation simple des résultats (trois paramètres : temps, densité spectrale, fréquence/vélocité), les travaux s'orientent vers une quantification des résultats en s'efforçant de relier précisément les caractéristiques spectrales à celle de l'écoulement $[12,15,16]$.

\section{Capteurs}

Les capteurs utilisés en vélocimétrie Doppler, bien que parfois moins élaborés que ceux utilisés en échographie présentent naturellement un certain nombre de caractéristiques communes, auxquelles s'adjoignent des spécifications propres au domaine d'utilisation, en particulier dimensionnelles. L'élément transducteur électromécanique est constitué par une pastille de céramique ferro-électrique (en général du zircotitanate de plomb). $\mathrm{La}$ configuration du capteur dépend naturellement $\mathrm{du}$ mode d'utilisation.

Le recueil des vélocités peut s'effectuer:

- en approche périvasculaire, pour l'expérimentation et l'usage per-opératoire,

- en approche intravasculaire (cathétérisme vélocimétrique peu répandu),

- en approche transcutanée, largement utilisée pour les examens cliniques non traumatisants.

L'obtention de mesures quantitatives pose le problème de l'angulation. Il est important de souligner que la vélocité réellement mesurée avec un capteur Doppler est la composante de la vélocité liquidienne selon l'axe du faisceau ultrasonore. Si l'on connaît l'angle $\theta$ entre le faisceau ultrasonore et les filets liquidiens, on peut écrire :

$$
v=c F_{D} / 2 F_{e} \cos \theta=K_{v} F_{D}\left(c / 2 F_{e}\right)
$$

et étalonner les fréquences Doppler en termes de vélocités.
Une imprécision sur la connaissance de l'angle $\theta$ donne une imprécision sur la vélocité mesurée. On peut exprimer l'erreur relative sur l'étalonnage en fonction de l'imprécision de positionnement du capteur (pour de faibles écarts) :

$$
\partial K_{v} / K_{v}=-\operatorname{tg} \theta \partial \theta
$$

L'erreur commise est d'autant plus importante que l'angulation du faisceau est grande.

Lors des mesures en condition périvasculaire et dans certains cas de mesures en bout de cathéter, il est parfois possible de connaître avec une précision suffisante l'angle $\theta$; ce n'est plus le cas pour les mesures transcutanées pour lesquelles le relevé de la direction du vaisseau peut être très délicate à effectuer. On peut avoir une estimation de l'angle $\theta$ en faisant toumer le capteur de cet angle à partir de la position où l'effet Doppler est nul, mais cette dernière est mal définie. De préférence, on utilisera, lorsque plusieurs incidences d'exploration du vaisseau sont possibles, une méthode de triangulation ou de biangulation. Il est parfois possible, pour réaliser une biangulation, de disposer deux transducteurs inclinés symétriquement dans le même corps de capteur (cf. Figure 5) [11, 15]. A l'intersection des faisceaux ultrasonores, on peut écrire :

composante longitudinale de la vélocité : $\nu_{1}-\nu_{2} / 2 \cos \theta$ composante transversale de la vélocité : $\nu_{1}+\nu_{2} / 2 \sin \theta$

$v_{1}$ et $v_{2}$ étant les composantes de vélocité relevées séparément par les deux transducteurs dans leur direction.

Dans le voisinage du volume d'intersection des faisceaux, on peut considérer les relations ci-dessus comme valables en première approximation pour des points homologues. Un tel système permet donc de reconstituer les composantes longitudinales et radiales de la vélocité. Il permet par ailleurs, pour la composante longitudinale, de réduire considérablement l'erreur due à l'imprécision de positionnement angulaire du capteur.

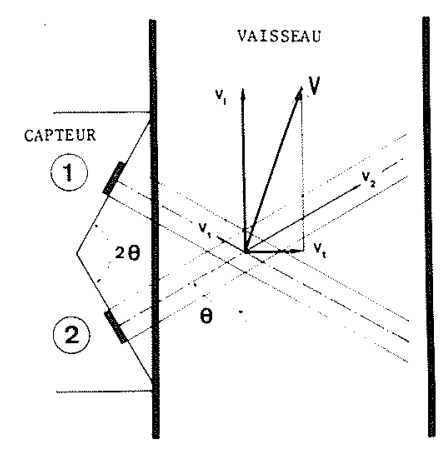

Figure 5 - Principe d'une mesure avec biangulation

\section{Applications}

Les applications de la vélocimétrie Doppler sont très nombreuses. On peut schématiquement les classer en cinq groupes : vélocimétrie et débitmétrie dans les vaisseaux périphériques, vélocimétrie dans les vaisseaux profonds et les cavités cardiaques, imagerie vasculaire, vélocimétrie de structures et applications expérimentales. 
Vélocimétrie et débitmétrie dans les vaisseaux périphériques

Un grand nombre de sites sont accessibles par voie transcutanée tant pour le réseau artériel que pour le système veineux. Usuellement, on peut explorer les artères suivantes :

- au niveau de la circulation des membres : sousclavière, axilaire, humérale, radiale, cubitale, digitale pour les membres supérieurs ; fémorales communes et superficielles, poplitées, tibiales postérieure et pédieuse pour les membres inférieurs;

- au niveau de la circulation cérébrale : carotides primitives, internes et externes, vertébrales et ophtalmiques.

On peut explorer le système veineux aux mêmes niveaux. A ces différents sites de mesure on pourra effectuer généralement des relevés de vélocité, calibrés ou non, et parfois des mesures de débit.

\section{Relevés de vélocité}

Ces relevés peuvent être effectués à l'aide d'appareils à émission continue ou pulsée. Ces derniers peuvent aider à différencier des vaisseaux proches 1'un de l'autre et apporter des renseignements intéressants sur la distribution des vélocités.

Même sans étalonnage rigoureux, un grand nombre de troubles vasculaires ou de cardiopathies à retentissement vasculaire peuvent être détectés. Il est hors de propos de dresser un tableau de la pathologie vasculaire et de la séméiologie des courbes Doppler correspondantes et, de plus, les travaux publiés dans la littérature sont très nombreux. On peut dire cependant que les éléments permettant le diagnostic sont généralement obtenus à partir des caractéristiques de la courbe de vélocité enregistrée pour différentes positions du capteur le long du segment vasculaire examiné (cf. Fig. 6) : valeurs crête de la vélocité pendant la systole $\left(V_{c s}\right)$, valeur crête du flot rétrograde éventuel $\left(V_{c r}\right)$, de la composante continue $\left(V_{m}\right)$ et surtout valeurs relatives de ces divers éléments. Par exemple, la pulsatilité de l'écoulement est reflétée par le rapport $V_{c s} / V_{m}$ et sa diminution révèle la présence d'un frein circulatoire en amont. Le rapport $V_{c r} / V_{c s}$ ou le rapport des aires correspondant aux flux négatif et positif s'il s'avère trop important au niveau d'une sous-clavière ou même d'une fémorale indiquera la présence d'une régurgitation de la valve aortique. Une valeur anormalement basse du temps de montée de la courbe de vélocité (angle $\beta$ ) pourra faire suspecter un rétrécissement de l'orifice

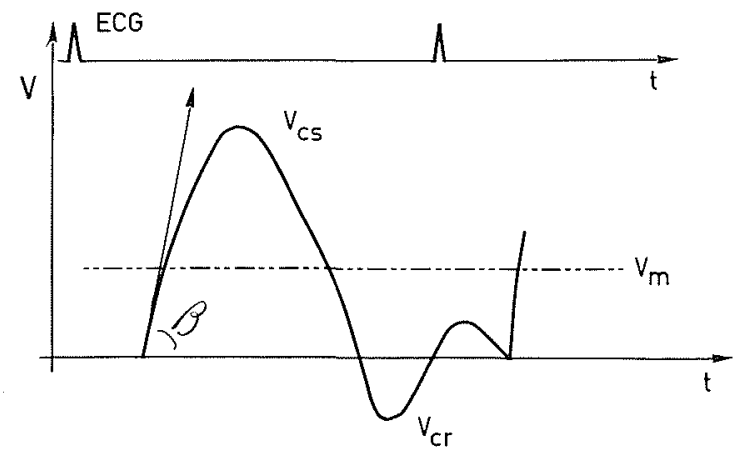

Figure 6 -Eléments caractéristiques d'une forme d'onde de vélocité. valvulaire aortique. La chronologie des divers éléments de la forme d'onde (par rapport à l'électrocardiogramme), la présence d'une inversion éventuelle du flux principal par rapport à sa direction normale, seront également des indicateurs importants.

A titre d'exemple encore, un des objectifs les plus fréquents lors d'un examen Doppler est la recherche des oblitérations vasculaires. En déplaçant la sonde le long du segment vasculaire suspecté, et en gardant une angulation à peu près constante, on enregistre les formes d'ondes aux diverses positions. La figure 7 schématise ce qui peut s'observer au niveau d'une sténose. En amont, la forme d'onde de vélocité est normale ; en aval, la pulsatilité est réduite et ce dautant plus que le rétrécissement est sévère ; au droit même de la sténose, on note un accroissement de la vélocité dû à la diminution de la section vasculaire et juste derrière le rétrécissement, la présence de tourbillons, de turbulence pourra être perçue sur la forme d'onde par ses irrégularités et ses instabilités d'un cycle pulsatile à l'autre, mais aussi par l'écoute du signal Doppler et naturellement par son analyse spectrale.

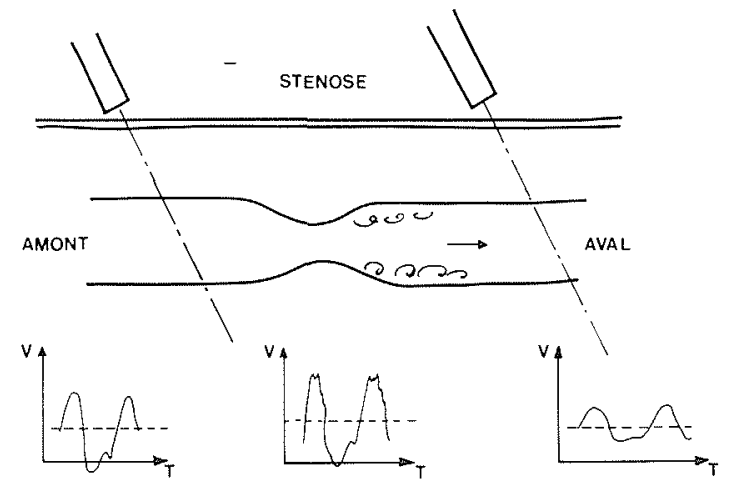

Figure 7 - Principe de l'observation d'une sténose vasculaire

\section{Mesure de la vélocité et du débit}

Des mesures calibrées de vélocité et de débit sont accessibles par les appareils à émission pulsée aux sites où la méthode de biangulation est applicable [17]. C'est le cas lors des mesures per-opératoires où des débits d'organes ou de revascularisation après chirurgie réparatrice pourront être mesurés. C'est également possible au cours d'un examen transcutané lorsqu'un petit segment vasculaire est accessible sous deux incidences, ce qui est assez souvent le cas pour les vaisseaux périphériques. Un exemple particulier d'application de la mesure transcutanée du débit est donné par les shunts artério-veineux. sous-cutanés des hémodialysés chroniques dans lesquels le contrôle du débit est un élément important de surveillance et aisément réalisable.

Vélocimétrie dans les vaisseaux profonds et les cavités cardiaques

L'écoulement du sang dans l'aorte initiale, l'aorte horizontale, l'artère pulmonaire, les by-pass chirurgicaux aorto-coronaires, les valves cardiaques sont accessibles lors d'explorations transcutanées, réalisées, 
sauf exception, par les appareils à émission pulsée où la discrimination en distance est nécessaire pour éliminer l'interférence des vaisseaux superficiels $[18,19,20,21]$. Il existe peu de fenetres acoustiques en direction de ces sites thoraciques en raison de l'écran introduit par l'air des poumons ; le capteur est en général posé soit dans l'encoche suprasternale, soit au droit d'un espace intercostal. Ce petit nombre de fenêtres acoustiques disponibles rend extrêmement difficile, encore à l'heure actuelle, les mesures quantitatives de vélocité et de débit dans les vaisseaux thoraciques profonds.

\section{Imagerie vasculaire}

Les systèmes échographiques et Doppler à émission continue ou pulsée peuvent être associés pour donner une image de la lumière vasculaire sur un territoire déterminé. Comme pour un appareil échographique, la position du capteur est recopiée sur l'écran d'un oscilloscope (Fig. 8) et une présentation de type B est faite en remplaçant l'information sur l'amplitude des échos reçus par une information Doppler : si un écoulement existe en un point donné, l'effet Doppler résultant est détecté et éclaire le spot [23]. En déplaçant le capteur, une cartographie de la lumière vasculaire peut être ainsi décrite sur l'écran et révéler la présence d'oblitérations. Des développements techniques sont encore nécessaires pour obtenir rapidement une image douée d'une résolution spatiale et d'une fiabilité suffisantes.
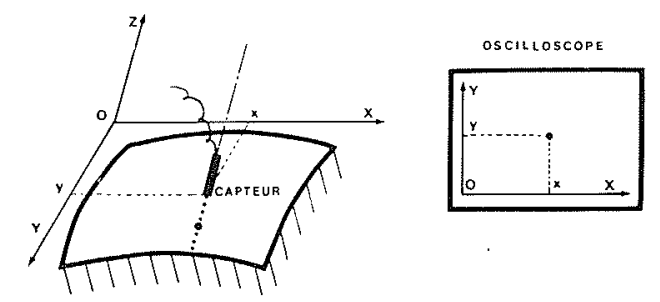

Figure 8 - Principe de l'imagerie vasculaire Doppler.

\section{Vélocimétrie de structures}

Les techniques Doppler sont parfois utilisées pour obtenir directement une mesure de la vélocité de déplacement d'une structure déterminée comme le cœur foetal ou les parois ventriculaires cardiaques [23].

\section{Applications à l'expérimentation}

De nombreuses possibilités pour les études hémodynamiques en expérimentation animale ont été ouvertes par les techniques Doppler, et en particulier par la méthode Doppler à émission pulsée. Quelques remarques peuvent être faites :

- les conditions de mesure sur l'animal sont très proches des conditions per-opératoires chez le patient. Lorsque les objectifs et les résultats souhaités sont clairement définis par l'expérimentation, les procédures mises au point sont aisément applicables au domaine chirurgical ;

- les conditions expérimentales permettent de réaliser des études hémodynamiques fondamentales. Les résultats de certaines d'entre elles peuvent être utilisés. en clinique chirurgicale ou en diagnostic transcutané ;

- les caractéristiques très complexes de l'écoulement du sang dans les grands vaisseaux rendent souvent une approche mathématique ou une approche systématique in vivo difficile, et des expérimentations in vitro peuvent aider à l'acquisition des lois de comportement.

Le réseau veineux, le système artériel et l'écoulement au travers des valves naturelles ou prothétiques peuvent être étudiés.

Concernant l'écoulement veineux, des configurations anatomiques rendent parfois complexe la forme de l'écoulement et une étude précise de ses caractéristiques apparaît nécessaire. La figure 9 en donne un exemple. Il s'agit de la distribution de la vélocité moyenne relevée au

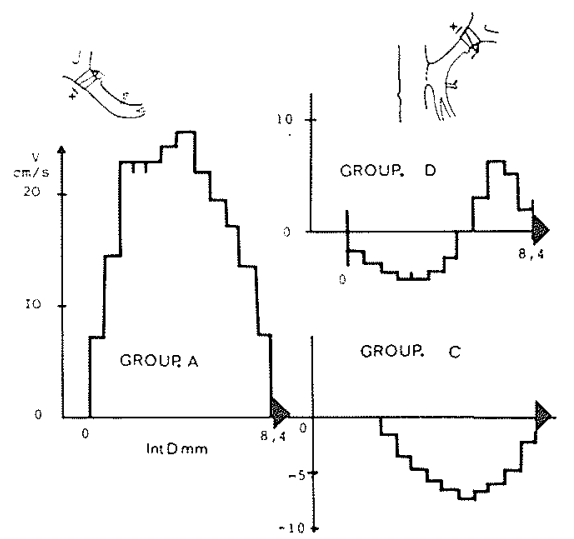

Figure 9 - Modification du profil de vélocité moyenne dans la veine porte, avant $(A)$ et en aval d'une anastomose porto-cave chez le chien normal (B) et après ligature des veines sushépatiques (réf. 24)

niveau de la veine porte, avant $(\mathrm{A})$ et après réalisation d'une anastomose latéro-latérale avec la veine cave (en B et C) [24]. Le profil des vélocités affecte une forme en dôme dans la veine porte normale. Après l'ouverture de l'anastomose portocave, le profil devient bidirectionnel (B) : à proximité du capteur ultrasonore, dans la partie gauche de la veine porte, les vélocités sont dirigées vers le foie alors que dans la partie droite, elles sont dirigées du foie vers l'anastomose. La composante d'écoulement vers le foie se trouve supprimée par la ligature des veines sus-hépatiques (C).

A titre d'exemple également d'études réalisées à propos de valves artificielles, la figure 10 rapporte une étude effectuée juste en aval d'une valve de Starr in vitro. Une série de profils de la vélocité instantanée a été mesurée pour différents instants pendant le cycle de pompe. Au début de l'éjection, tous les filets liquidiens ont à peu près la même vélocité. Une encoche centrale apparaît ensuite correspondant à l'arrêt de la bille, et les vélocités centrales négatives révèlent ensuite son mou- 
vement de retour. Le long palier diastolique où les vélocités sont pratiquement nulles démontre la bonne fermeture de la valve.

Concernant le réseau artériel, des travaux récents ont montré l'influence des facteurs mécaniques de l'écoulement du sang sur la genèse de l'athérosclérose. La croissance des dépôts athéromateux intervient en des sites privilégiés des artères, comme par exemple le long de la paroi intérieure d'une courbe ou de la lèvre proximale d'une branche. Le mécanisme réel de la croissance des plaques n'est pas actuellement clairement établi, mais il est évident que des données hydrodynamiques précises sont nécessaires.

Pour illustrer un type de mesure rendu possible par les techniques Doppler à émission pulsée, la figure 11 présente un exemple de distributions de la vélocité instantanée relevées au niveau de l'arche aortique du chien. A l'aide de capteurs doubles permettant une biangulation, les vecteurs vélocité ont été mesurés dans le plan de courbure de l'arche (positions 2, 3, 5,6 et 7) et selon des diamètres perpendiculaires à ce plan (positions 1, 4 et 8). L'évolution complète au cours du cycle cardiaque de ces distributions a été étudiée [15, 25], mais ne sont représentées sur la figure que la répartition des vélocités correspondant à la crête de l'éjection

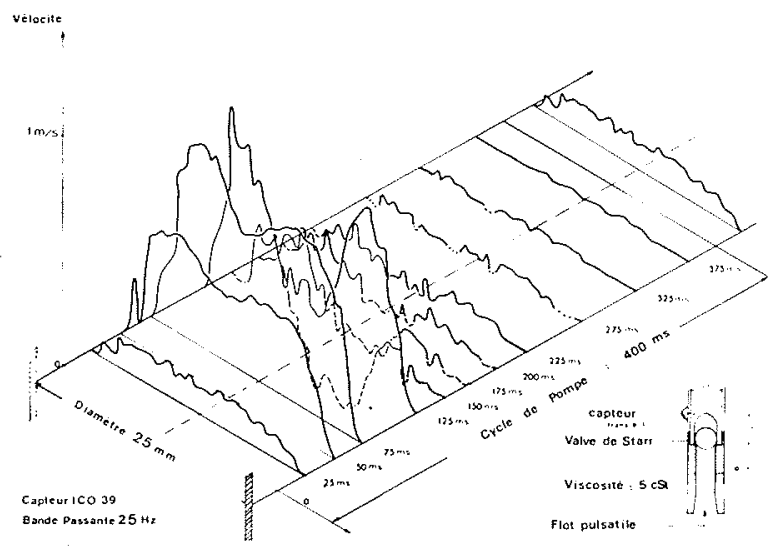

Figure 10 - Profils de vélocité en aval d'une valve de Starr (variations relevées in vitro pendant un cycle de pompe)

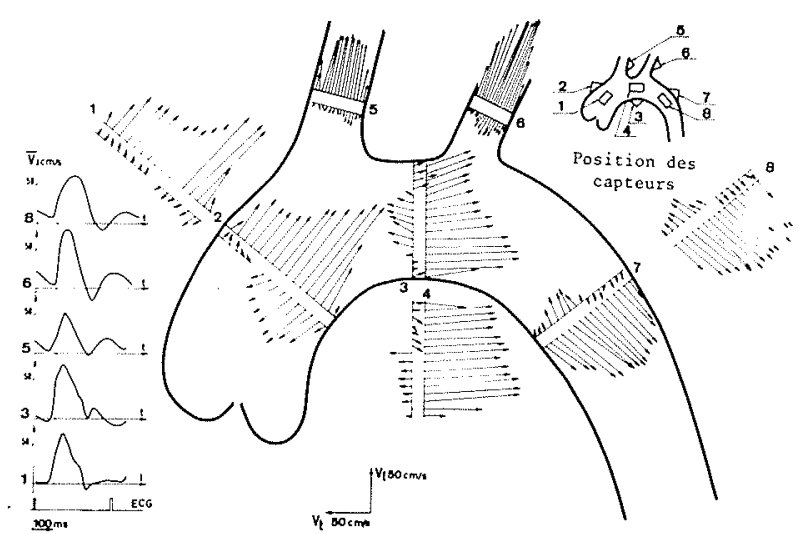

Figure 11 - Distribution de la vélocité instantanée le long de l'arche aortique à l'instant des débits crêtes positif et rétrograde (réf. 25). systolique et au maximum du flot rétrograde. L'évolution des débits calculés par intégration semi-annulaire des profils de vélocité, est représentée sur la partie gauche de la figure. D'une façon générale, l'étude de ces distributions de vélocité montre que, au niveau de l'aorte initiale, la couche limite semble d'épaisseur relativement faible et les forces d'inertie paraissent prépondérantes pour l'écoulement. Au niveau de l'aorte descendante et des troncs, par contre, le profil se régularise et l'influence de la viscosité paraît nettement plus marquée. A partir des profils de vélocité mesurés notamment près des parois vasculaires, le gradient radial de vélocité, le frottement pariétal et un nombre de Reynolds caractérisant l'écoulement crête peuvent être estimés $[12,15]$.

\section{Conclusion}

$\mathrm{Au}$ cours de cette brève présentation, nous avons décrit le principe de la vélocimétrie ultrasonore Doppler et les principaux systèmes de mesure couramment utilisés actuellement. Des progrès sont encore à faire concernant par exemple la sensibilité, la résolution et la quantification de l'analyse spectrale, mais telles quelles, les techniques Doppler vélocimétriques offrent un grand nombre de possibilités aussi bien pour l'expérimentation que pour le diagnostic non traumatisant.

\section{Références bibliographiques}

[1] FRANKLIN D.L., SCHLEGEL W.C. et RUSHMER R.F. - Blood flow measured by Doppler frequency shift of back-scattered ultrasound, Science, 1961, 134, 564 . 565.

[2] SATOMURA S. - Study of flow patterns in peripheral arteries by ultrasonics, $\boldsymbol{J}$. Acoust. Soc. Japan, 1959, 15, 151-158.

[3] Mc LEOD F.D. - A directional Doppler flowmeter in Digest of the 7 th Intern. Conf. on Med. and Biol. Engng., 1967, p. 196-217, Stockolm.

[4] CHICHE P., KALMANSON D., VEYRAT C. et TOUTAIN G. - Enregistrement transcutané du flux artériel par fluxmètre directionnel à effet Doppler. Description d'un appareillage et premiers résultats, Soc. Med. Hôp. Рaris, 1968, 119 (1), 87-95.

[5] CATHIGNOL D., FOURCADE C., GUILlaUd O., GOUTTE R. et DESCOTES J. - Description d'un nouveau débitmètre sanguin directionnel à effet Doppler, Presse Med. 1970, 78 (4), 189-190.

[6] POURCELOT L. - Nouveau débitmètre sanguin à effet Doppler in "Premier Congrès Mondial sur le Diagnostic Ultrasonore en Médecine", 1969, Vienne.

[7] PERONNEAU P. et LEGER F. - Doppler ultrasonic pulsed blood flowmeter in "The 8th Intern. Conf. on Med. and Biol. Engng., 1969, pp. 10-11, Chicago.

[8] BAKER D.W. - Pulsed ultrasonic Doppler blood-flow sensing. IEEE Transactions on sonics and ultrasonics, $1970,17,170-185$.

[9] PERONNEAU P., LEGER F., HINGLAIS J., PELLET M. et SCHWARTZ P.Y, - Vélocimètre sanguin à effet Doppler à émission ultrasonore pulsée, $L$ 'Onde Electrique, 1970, 50, 369-389.

[10] Mc LEOD F.D. - Multichannel pulse Doppler techniques, in "Cardiovascular Applications of Ultrasound", R.S. Reneman Ed., North Holland Publ. Co. Amsterdam, 1974 , p. 85 . 
[11] PERONNEAU P., BOURNAT J.P., BUGNON A., BARBET A. et XHAARD M. - Theoretical and practical aspects of pulsed Doppler flowmetry : real-time application to the measure of instantaneous velocity profiles in vitro and in vivo in "Cardiovascular Applications of Ultrasound", Reneman R.S. Ed., 1974, p. 66, North Holl. Publi. Co., Amsterdam.

[12] PERONNEAU P., SANDMANN W. et XHAARD M. Blood flow patterns in large arteries in "Ultrasound in Medicine", White D.N. and Brown R.E. Eds., 1977, Vol. 3B, p. 1 193, Plenum Press Publishing, New-York.

[13] BOURNAT J.P. - Mesure des fréquences Doppler in "Vélocimétrie Doppler Ultrasonore - Application à l'étude de l'écoulement sanguin dans les gros vaisseaux", Péronneau P. Ed., 1974, Vol. 34, pp. 115-122, INSERM', Paris.

[14] SIGEL B., FELIX W.R., POPKY G.L. et IPSEN J. Diagnosis of lower limit venous thrombosis by Doppler ultrasound techniques, Archives of Surgery, 1972, 104, 174-179.

[15] PERONNEAU P. - Analyse de l'écoulement sanguin dans les gros vaisseaux par méthode ultrasonore, Thèse de Doctorat d'Etat, mai 1977, Orsay.

[16] SANDMANN W., PERONNEAU P., UlRich B., BOURNAT J.P., XHAARD M. et GISBERTZ K.H. - Die Messung von Turbulenz mit Ultraschall-DopplerVerfahren am Strömungsmodell, am Hund am Menschen in "Hypertonie-Risikfactor in der Angiologie", Zeitier E. Ed,, Verlag Gerhand Witzstrock, 1976, Köln, pp. 222229.

[17] PERONNEAU P., XHAARD M., DIEBOLD B., FIESSINGER J.N., LEVY B. et BOURQUELOT P. Débitmétrie transcutanée par vélocimétrie ultrasonore Doppler à émission pulsée, La Nouvelle Presse Médicale, $1976,5,2547$.
[18] BAKER D.W. et JOHNSON S.L. - Doppler echocardiography in "Cardiac Ultrasound", Gramiak R. Ed., 1975, Mosly C.V. Publ., St Louis.

[19] DIEBOLD B., GUERMONPREZ J.L., PERONNEAU P., BARBET A., BOURASSA M.G. et THEROUX P. - Noninvasive assessment of the patency of aorto-coronary grafits by pulsed Doppler velocimetry. A preliminary study. in "Int. Conf. on Cariovascular Dynamics", 1976, Philadelphie, U.S.A.

[20] KALMANSON D., VEYRAT C., BOUCHAREINE F. et DEGROOTE A. - Non-invasive recording of mitral valve flow velocity patterns using pulsed Doppler echocardiography, British Heart Journal, may 1977, 39 (5), 517-528.

[21] LIGHT L.H. - Transcutaneous aortovelography - a new window on the circulation ?, British Heart J., 1976, 38, 433.

[22] BOURNAT J.P. - Visualisation vasculaire in "Vélocimétrie Doppler Ultrasonore - Application à l'étude de l'écoulement sanguin dans les gros vaisseaux", Péronneau P. Ed., 1974, Vol. 34, pp. 115-122, INSERM, Paris.

[23] GUGLIELMI J.P., PERONNEAU P., DIEBOLD B., LEFORT J.F., PERNOD J. - Real-time Doppler detection of heart wall contraction speed, in "Ultrasound in Medicine" White D.N. and Brown R.E. Eds., 1977, Vol 3B, p. 1193, Plenum Press Publishing, New-York.

[24] LOISANCE D., PERONNEAU P., PELLET M., LENRIOT J.P. - Hepatic circulation after side to side portacaval shunt in dogs. Velocity patterns and flow rate changes studied by an ultrasonic velocimeter, Surgery, 1973, 73. (1), 43-52.

[25] PERONNEAU P., HINGlais J., XHAARD M., DELOUCHE P., PHILIPPO J. - The effects of curvature and stenosis on pulsatile flow in vivo and in vitro in "Cardiovascular Applications of Ultrasound", Reneman R.S. Ed., 1974, Vol. 1, pp. 66-84, North Holland Publishing Co., Amsterdam.

\section{Discussion}

\section{Président : M.Y. JAFFRIN}

M. le Président remercie M. PERONNEAU de son exposé très clair et très intéressant.

Amorçant lui-même la discussion, il remarque l'importance, pour la précision de la mesure, de la réduction du volume de mesure. En vélocimétrie ultrasonore, ce problème est résolu partiellement par focalisation du faisceau.

M. PERONNEAU précise qu'en procédure ultrasonore, on peut réduire considérablement les dimensions latérales du faisceau jusqu'à quelque chose de l'ordre du millimètre.

Par contre la réduction du volume de mesure dans la direction du faisceau pose des problèmes de bande passante, de capteurs et de sensibilité liés à l'atténuation importante de l'onde acoustique à travers les tissus.

M. LEDUCQ demande quelle précision on peut espérer pour une mesure par biangulation sur un vaisseau superficiel comme, par exemple, une artère humérale.

M. PERonNeAu indique que cela dépend toujours un peu des conditions géométriques et de l'expérience de l'opérateur, mais que l'on peut espérer une précision de $10 \%$ à $15 \%$. Il cite à cet égard le cas des shunts hémodyalisés sous-cutanés dont il faut bien connaître et surveiller le débit : la méthode ultrasonore est la seule méthode externe qui le permette avec une précision voisine de $10 \%$ ou même meilleure.

Le Président, observant que le faisceau est dans certains cas plus large que le vaisseau, đemande à $M$. PERONNEAU comment la vitesse moyenne mesurée par détection du passage par zéro diffère de la vitesse moyenne dans le vaisseau.
M. PERONNEAU répond que le compteur de passage par zéro donne la fréquence quadratique moyenne dont la différence avec la fréquence moyenne est notable et dépend beaucoup de la configuration du profil d'écoulement. Celle-ci peut introduire des écarts de 15-20 ou même $30 \%$.

Empiriquement, précise-t-il, il semble que plus le volume de mesure est important, plus le vaisseau est gros et plus la réponse du "zéto crossing" s'écarte de la vélocité moyenne. Il mentionne également parmi les causes de distorsion l'atténuation de l'onde au travers du sang lorsque la profondeur de la mesure s'étage sur un ou deux centimètres: vis à vis du détecteur, les globules les plus éloignées n'ont pas le même poids que les globules les plus proches.

En réponse à une question de $M$. LEDUCQ, $M$. PERONNEAU indique ensuite que la méthode Doppler ne lui paraît pas la mieux indiquée pour effectuer une mesure quantitative de la déformation de la paroi vasculaire dans le cas d'un vaisseau superficiel.

M. REMENIERAS remarque que pour la mécanique des fluides, l'inversion des vitesses sur un même profil ne se limite pas au cas des circuits courbes; elle se produit également dans des tubes droits. C'est ainsi que la vitesse peut être de sens inverse au droit de la paroi. Il rappelle les études menées à bien il y a plusieurs années par M. VALEMBOIS sur les courants alternatifs pour lesquels ces problèmes d'inversion doivent tout spécialement retenir l'attention. 